\title{
Multi-messenger physics opportunities with core-collapse supernovae
}

\section{Shunsaku Horiuchi*}

Center for Neutrino Physics, Department of Physics, Virginia Tech, Blacksburg, VA 24061, USA

E-mail: horiuchi@vt.edu

\begin{abstract}
The multi-messenger signatures - in neutrinos, gravitational waves, and electromagnetic emission-from a core-collapse supernova, and their synergies, are reviewed. Within the Milky Way, neutrinos offer an excellent alert, providing both temporal and angular information to facilitate the detection of electromagnetic and gravitational waves. Beyond the Milky Way, corecollapse supernovae hosting relativistic jets are potential sources of ultra-high energy cosmic rays and high-energy neutrinos that can explain observations by Auger and IceCube, respectively.
\end{abstract}

Neutrino Oscillation Workshop (NOW2018)

9 - 16 September, 2018

Rosa Marina (Ostuni, Brindisi, Italy)

\footnotetext{
*Speaker.
} 


\section{Introduction}

With the recent detections of extraterrestrial neutrinos and gravitational waves, we have entered the era of multi-messenger astronomy. One exciting target of the multi-messenger approach is the core-collapse supernova. A massive star (with zero-age main sequence mass above some $8 M_{\odot}$ ) will undergo core collapse at the end of its life, triggering an optical supernova explosion. The collapse coincides with the emission of a burst of neutrinos (of energies of order tens of $\mathrm{MeV}$ ) and gravitational waves (at frequencies of $10^{2}-10^{3} \mathrm{~Hz}$ ) lasting several tens of seconds. A future nearby core-collapse supernova will offer the unprecedented and scientifically rich opportunity to detect three cosmic messengers-electromagnetic emission, neutrinos, and gravitational waves-from a common source.

Figure 1 shows an example from Ref. [1] of the expected time sequence for a core-collapse's multi-messenger signals, starting from before (left panel) and after (right panel) core bounce, for a non-rotating solar metallically star of $17 M_{\odot}$ initial mass. Prior to collapse, the neutrino emission during the last stages of silicon burning (labeled "Pre-SN") rise, until being overtaken by the neutrino burst emitted by the collapsing core. Gravitational waves are emitted from soon after core bounce. The electromagnetic signal remains unchanged (labeled "progenitor") until the supernova shock breaks through the stellar photosphere and causes the shock breakout emission (labeled "SBO"). The SBO is followed by the plateau and decay phases. Finally, the supernova sources cosmic rays during its remnant phase or association with a gamma-ray burst (not shown).

Among the multi-messenger emissions, neutrinos play a uniquely key role. They dominate the energetics, i.e., they carry away the dominant fraction of the gravitational binding energy liberated by the core collapse, a staggering $\sim 3 \times 10^{53} \mathrm{erg}$. For many progenitors, neutrinos are thought to drive the supernova explosion by transferring energy and reviving the stalled bounce shock. Although such neutrino-driven explosions have been difficult to obtain in spherically symmetric simulations, recent studies have shown the crucial roles played by hydrodynamic instabilities for the explosion mechanism. Successful explosions have recently been obtained with self-consistent first-principle simulations in three spatial dimensions (see, e.g., [2] for a review). Neutrinos also determine the proton to neutron ratios of neutrino-driven winds, affecting nucleosynthesis.

Recent studies have revealed the importance of supernova diversity. In these studies, spherically symmetric or axisymmetric simulations are used, which allow hundreds of initial conditions to be simulated. They have unraveled novel insights, among them that explosions come in pockets of parameter space rather than continuous. Ref. [3] defined the compactness parameter,

$$
\xi_{M}=\left.\frac{M / M_{\odot}}{R(M) / 1000 \mathrm{~km}}\right|_{t}
$$

to provide a simple one-parameter characterization of the stellar core density structure that associates with the rate of mass accretion onto the protoneutron star. Using parameterized spherical symmetric simulations, Ref. [3] showed that high compactness progenitors are conducive to prompt black hole formation (i.e., without a supernova; sometimes called "failed supernova"). By strategically adopting an appropriate mass $M$, the compactness can in fact capture the mass accretion during the critical epoch of shock revival, and become a reasonable metric of whether the star explodes or fails to explode. In fact, a single compactness parameter can correctly predict the 


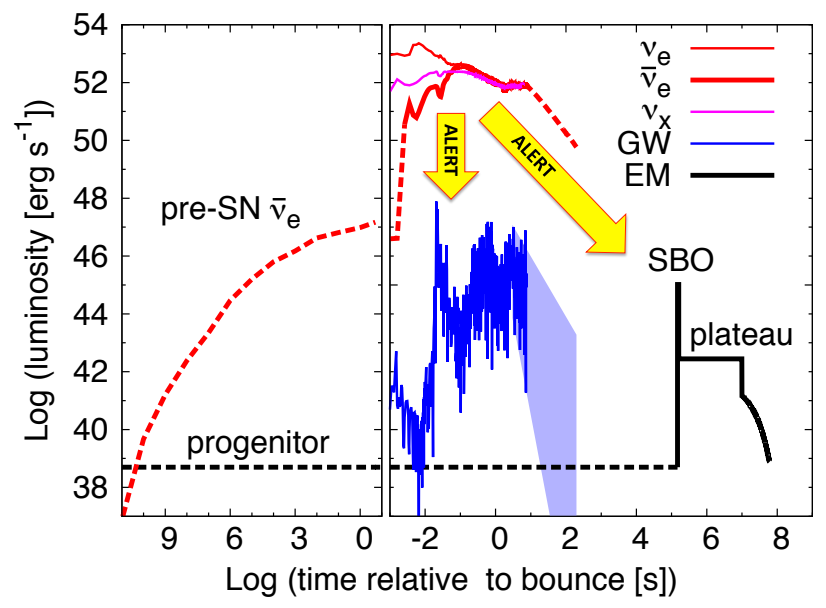

Figure 1: The time sequence of multi-messenger signals: pre- (left panel) and post- (right panel) core bounce of a non-rotating $17 M_{\odot}$ progenitor. Neutrinos $\left(v_{e}, \bar{v}_{e}\right.$, and $v_{x}$ are shown by red, thick red, and magenta, respectively, where $v_{x}$ represents heavy-lepton neutrinos $v_{\mu}, v_{\tau}, \bar{v}_{\mu}$, and $\bar{v}_{\tau}$ ), gravitational waves (blue), and electromagnetic signals (black) are shown. Solid lines are predictions from an axis-symmetric hydrodynamical simulation, while dashed lines are approximate predictions. The electromagnetic signal exhibits the shock breakout (SBO), plateau, and decay components. At even later epochs, the supernova generates cosmic rays and high-energy neutrinos (not shown). Note that the height of the curves do not reflect the energy output in each messenger; the total energy emitted in the form of $\bar{v}_{e}$, photons, and gravitational waves are $\sim 6 \times 10^{52} \mathrm{erg}, \sim 4 \times 10^{49} \mathrm{erg}$, and $\sim 7 \times 10^{46} \mathrm{erg}$, respectively. From Ref. [1]

outcome of core collapse in as high as $88 \%$ of massive stars [4]; extending to a two-parameter criterion, the predictability improves to $97 \%$ [5]. This is impressive, considering the compactness is a simplistic one-parameter attempt to describe an inherently multi-dimensional and time evolving phenomenon. Quantitatively, recent works show $\xi_{2.5} \sim 0.3$ to be the divide between successful explosions and collapse to black holes, although this has yet to be shown with three-dimensional simulations and uncertainties remain large $[5,6]$. Based on present suites of single star evolution, about $15-20 \%$ of stars with mass $>8 M_{\odot}$ have compactness $\xi_{2.5}>0.3$, suggesting a non-negligible fraction of collapse to black holes. Interestingly, similarly high values of failed supernovae are also suggested from several different astronomical observations. These are: (1) the red supergiant problem, (2) the black hole mass function, (3) the cosmic supernova rate, and (4) the Survey about Nothing. While each of them have other potential solutions, it is interesting to note that all can be explained by massive stars failing to produce supernovae above $\xi_{2.5} \sim 0.2-0.3[7,8]$, which is also close to the criterion suggested by simulations. More work, both theoretical and observational, are needed to unravel the physics of core collapse and their diversity. Multi-messenger observations have the potential to uniquely improve our understanding of core-collapse supernovae.

\section{Multi-messenger: within the Milky Way galaxy}

The next core-collapse supernova in the Milky Way will offer a scientifically rich opportunity to detect all three cosmic messengers-electromagnetic, neutrinos, and gravitational waves-from a common source. In this context, the neutrino is a compelling trigger as well as messenger. 
- Neutrinos will reveal $I F$ followup should observe. Namely, the core-collapse neutrino burst can be detected with current neutrino detectors with high significance from anywhere within the Milky Way galaxy. Super-Kamiokande will detect $\sim 7,000$ inverse-beta decay events from a core collapse at the Galactic center distance (often taken to be a nice round number of $10 \mathrm{kpc}$, although recent measurements indicate a value closer to $8.5 \mathrm{kpc}$ ). Since the background rate over the duration of the neutrino burst (tens of seconds) is so low, detection is essentially background free, and its detection horizon reaches out beyond the edge of the Milky Way galaxy. Similarly, while IceCube do not individually reconstruct MeV neutrinos, it has the ability to detect the neutrino burst as a coherent rise in their optical modules' noise rates. Given IceCube's large volume and low-noise optical modules, IceCube is sensitive to a core-collapse neutrino burst from anywhere within the Milky Way galaxy. No other messenger provides such a compelling trigger.

- Neutrinos will reveal WHEN followup should observe. IceCube's high statistical detection allows exquisite sensitivity to the neutrino light curve. By fitting to the rise of the neutrino burst signal, IceCube can reconstruct the bounce time to within several milliseconds (e.g., $\pm 3.5 \mathrm{~ms}$ at $95 \%$ C.L. at $10 \mathrm{kpc}$ [9]). The systematic offset between the reconstructed and true bounce times is several $\mathrm{ms}$, and depends on the neutrino flavor oscillation scenario. Super-Kamiokande will have a similar time measurement [10]. These timing information are crucial for the detection of gravitational waves. As an example, consider the case of a $17 M_{\odot}$ non-rotating progenitor system where the asymmetries of the collapse generate gravitational waves [1]. The detection horizon using a coherent network of four gravitationalwave detectors (advanced LIGO Hanford and Livingston, advanced Virgo, and KAGRA) at their geophysical locations is a few kpc. The peak signal-to-noise in the spectrogram is only $\sim 3.5$ at a distance of $8.5 \mathrm{kpc}$, arising from the prompt convection signal. However, with timing information from neutrinos, the peak signal-to-noise rises to $\sim 7$; this is coincident gravitational-wave detection $[10,1]$. The coincident detection of neutrinos and gravitational waves will allow many tests, including the weak equivalence principle.

- Neutrinos will reveal WHERE followup should observe. This is most relevant for the detection of the SBO. The duration of the SBO in the explosion of a red supergiant star is $\sim 10^{3}-10^{4}$ seconds, but this is reduced to $\sim 10^{2}$ seconds for the explosion of a blue supergiant, and a mere few seconds for a Wolf-Rayet star [11]. The latter are not rare; they comprise some quarter of nearby core-collapse supernovae. The field of view of relevant optical telescopes range from a few to tens of degrees in diameter, depending on the brightness of the supernova (which in turn depends on the supernova location). Thus, they need to be alerted where to point. Neutrinos provide pointing, by two complementary ways. First, by the forward-peaked scattering on electrons, $v e \rightarrow v e$, which will appear concentrated along the forward cone. Super-Kamiokande would collect some $\sim 300$ such events for a core collapse at the Galactic center, and yield a pointing accurate to some $\sim 6$ degrees. Super-Kamiokande is presently undergoing upgrades to add gadolinium salt to the water tank, which will tag inverse-beta decay on an event-by-event basis via delayed coincidence. The tagging efficiency target is $\sim 90 \%$, which would dramatically reduce the inverse-beta decay events and improve pointing to $\sim 3$ degrees (again, for the Galactic center). This improvement is crucial for reducing the pointing error to within the fields of view of large 4-8 
$\mathrm{m}$ class telescopes which is only a few degrees. The second pointing mechanism is to use the timing difference between multiple neutrino detectors and use triangulation. With current detectors spread across Earth, triangulation is predicted to yield similar pointing errors of a few to several degrees, depending on the location on the sky [12].

- Finally, trigger information should be released quickly enough that followup would have the best chance of detection. This is especially crucial for optical followup that need to act and point to the appropriate location. The delay time between the neutrino burst and the SBO signal (the first electromagnetic signal) varies by orders of magnitude depending on the progenitor: from $\sim 10^{5}-10^{6}$ seconds for a red supergiant down to $\sim 10^{2}$ seconds for a WolfRayet star [11]. Presently, multiple automated alerts are in place, including SNEWS and also from EGADS. However, only Super-Kamiokande has the ability to determine a good enough pointing, but since its alert is not automated, it may miss cases with short delays such as the collapse of a Wolf-Rayet star [13]. Triangulation may fill this need for rapid automated pointing, but needs to be implemented.

There is no shortage of physics that could be studied with a future multi-messenger observation of a core-collapse supernova. Only a few selected examples will be described here. Generally, neutrinos and gravitational waves give us a semi-direct view of the collapsing core, which would be crucial for the first quantitative test of the supernova mechanism. For example, simulations that are able to obtain explosions often do so by episodes of strong instability activity called the standing accretion shock instability (SASI), occurring alternatively with convection-dominated periods. SASI comes in both sloshing or spiral motions, and are characterized by large-amplitude unipolar or dipolar deformations whose orientation flips on time scales of milliseconds. The deformations leave imprints on the neutrino luminosities and energies, making it possible to detect with neutrino detectors. Figure 2 shows the light curves at IceCube and Hyper-Kamiokande for three different progenitors with multiple (left pane), single (center panel), and no (right panel) SASI episodes. Based on current simulations, SASI, if relevant for the explosion, should be detectable from the Galactic center distance [14].
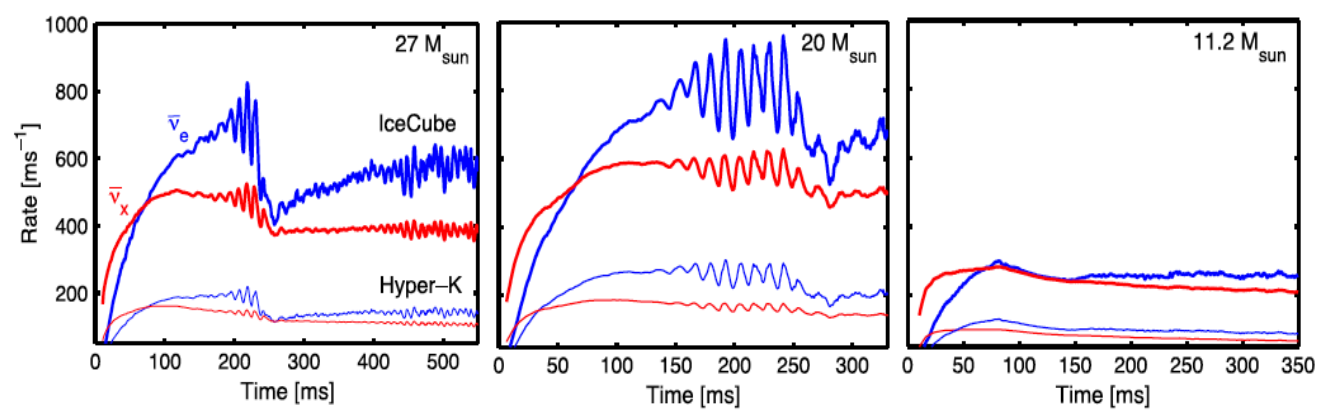

Figure 2: Neutrino detection rates at IceCube and Hyper-Kamiokande, as labeled, for $\bar{v}_{e}$ (blue) and $v_{x}$ assuming full swap by flavor conversion (red). Shown for three different progenitors: $27 M_{\odot}$ (left), 20M $\odot$ center, and $11.2 M_{\odot}$ (right). The $27 M_{\odot}$ progenitor has continued large mass accretion leading to multiple SASI episodes, while the $11.2 M_{\odot}$ progenitor has a small compactness and explodes relatively quickly without a SASI episode. The observer direction is chosen to be close to the SASI plane where the signal modulation is strongest. All distances are $10 \mathrm{kpc}$. From Ref. [14]. 
Also, the combination of neutrinos and gravitational waves can reveal core rotation. One example with a unique signature is that of the lighthouse effect, where the spinning of strong neutrino emitting regions leads to quasi-periodic modulation in the neutrino signal. These regions can be sustained by the growth of the spiral flows triggered by the low- $T /|W|$ instability [15]. This scenario also predicts the same modulation in gravitational waves, but at twice the frequency. Also, the neutrinos can reveal the formation of a black hole, either promptly or by mass fallback after an explosion, provided the formation occurs during the first few tens of seconds. This will be observable as a rapid increase in $v_{x}$ energies followed by a step function-like decline in neutrino luminosity [16]. The neutrinos can also reveal the properties of the progenitor core density profile through the neutrino rise during the first few hundreds of milliseconds, allowing a test of the compactness-exploitability connection [17].

\section{Multi-messenger: beyond the Milky Way}

The detection horizon of $\mathrm{MeV}$ neutrinos currently does not reach much further beyond the Milky Way, but this will change with the construction of next-generation detectors such as HyperKamiokande. With a volume close to ten times Super-Kamiokande, the time-integrated total inverse beta event in the $18-30 \mathrm{MeV}$ energy bin for the non-rotating $17 M_{\odot}$ model is,

$$
N_{e^{+}} \simeq 4.0\left(\frac{D}{1 \mathrm{Mpc}}\right)^{-2}\left(\frac{M_{\mathrm{det}}}{260 \mathrm{kton}}\right) .
$$

To claim significance with such low event counts requires low and well-understood backgrounds. Scaling up from SK-II, two neutrino events within a 10 second window ("doublet") should occur by chance only once every decade or so [1]. However, single neutrino events cannot be claimed with similar confidence. This is where a multi-messenger approach would be helpful—but in the reserve, i.e., using optical signals to inform neutrino detections. For supernovae in nearby galaxies, the optical early light curve would like be serendipitously detected by large field of view telescopes such as ASAS-SN and/or amateurs who frequently observe nearby galaxies. These observations can be used to model the light curve and constrain the bounce time to within a few hours, depending on the availability of early optical data. Given a time window of a few hours, even single neutrinos can be associated with the supernova. The few Mpc distance regime is an interesting target, since a rapid rise in the distribution of galaxies-and hence expected supernova rate-occurs at 3-4 Mpc. For example, the cumulative core-collapse supernova rate within $4 \mathrm{Mpc}$, based on historical supernova discoveries, is $\sim 0.8$ per year. Even though the neutrino event rate would be low-a few neutrinos per supernova - an almost annual occurrence would provide a welcomed addition to waiting for the next Galactic supernova.

Core-collapse supernovae remain multi-messenger targets at distances beyond a few Mpc, by their association with gamma-ray bursts. Many studies have focused on the gamma-ray bursts' ability to accelerate protons and higher charged nuclei to ultra-high cosmic ray energies $\left(\sim 10^{20}\right.$ $\mathrm{eV}$ ). Such scenarios predict ample high-energy neutrinos, the details depending on the gamma-ray burst model. This begs the question of whether gamma-ray bursts can explain the diffuse PeV neutrinos detected by IceCube. However, IceCube's stacked analysis of gamma-ray bursts has produced null detections, constraining their contribution to the diffuse $\mathrm{PeV}$ neutrino flux to $<1 \%$ [18]. 
Low-luminosity or choked jets however remain a viable solution since they are not constrained by the stacked analyses. Another intriguing possibility is that low-luminosity jets or magneticallydominate jets hosted by supernova can source ultra-high energy cosmic rays (UHECR) with a rather heavy nuclei composition [19]. This is possible due to (1) lower luminosity causing less photodisintegration of stellar nuclei, and/or (2) explosive nucleosynthesis to nuclei. Predictions are able to explain the mass composition, energy spectrum, and lack of anisotropies of ultra-high energy cosmic rays [20]. While other candidates for UHECRs exist, it is hard to explain all observables, especially the mass composition; supernova jets offer a unique self-consistent solution.

\section{Conclusions}

Core-collapse supernovae are inherently a multi-messenger phenomenon, releasing neutrinos, gravitational waves, cosmic rays, as well as electromagnetic emission. At Milky Way distances, neutrinos will provide a compelling alert to realize the detection of these multi-messenger components and learn about the core collapse. Beyond the Milky Way, supernova can source ultra-high energy cosmic rays and high-energy neutrinos, and explain observations by Auger and IceCube.

\section{References}

[1] K. Nakamura, S. Horiuchi, et al., Mon. Not. Roy. Astron. Soc. 461 (2016) no.3, 3296

[2] H.-T. Janka, T. Melson and A. Summa, Ann. Rev. Nucl. Part. Sci. 66 (2016) 341

[3] E. O'Connor and C. D. Ott, Astrophys. J. 730 (2011) 70

[4] O. Pejcha and T. A. Thompson, Astrophys. J. 801, no. 2, 90 (2015)

[5] T. Ertl, H.-T. Janka, S. E. Woosley, T. Sukhbold and M. Ugliano, Astrophys. J. 818 (2016) no.2, 124

[6] K. Nakamura, T. Takiwaki, T. Kuroda and K. Kotake, Publ. Astron. Soc. Jap. 67 (2015) no.6, 107

[7] C. S. Kochanek, Astrophys. J. 785 (2014) 28

[8] S. Horiuchi, K. Nakamura, et al., Mon. Not. Roy. Astron. Soc. 445 (2014) L99

[9] F. Halzen and G. G. Raffelt, Phys. Rev. D 80 (2009) 087301

[10] G. Pagliaroli, F. Vissani, E. Coccia and W. Fulgione, Phys. Rev. Lett. 103 (2009) 031102

[11] M. D. Kistler, W. C. Haxton and H. Yuksel, Astrophys. J. 778 (2013) 81

[12] V. Brdar, M. Lindner and X. J. Xu, JCAP 1804 (2018) no.04, 025

[13] S. M. Adams, et al., Astrophys. J. 778 (2013) 164

[14] I. Tamborra, G. Raffelt, F. Hanke, H. T. Janka and B. Mueller, Phys. Rev. D 90 (2014) no.4, 045032

[15] T. Takiwaki and K. Kotake, Mon. Not. Roy. Astron. Soc. 475 (2018) no.1, L91

[16] J. F. Beacom, R. N. Boyd and A. Mezzacappa, Phys. Rev. D 63 (2001) 073011

[17] S. Horiuchi, K. Nakamura, T. Takiwaki and K. Kotake, J. Phys. G 44 (2017) no.11, 114001

[18] M. G. Aartsen et al. [IceCube Collaboration], Astrophys. J. 824 (2016) no.2, 115

[19] S. Horiuchi, K. Murase, K. Ioka and P. Meszaros, Astrophys. J. 753 (2012) 69

[20] B. T. Zhang, K. Murase, S. S. Kimura, S. Horiuchi and P. Meszaros, Phys. Rev. D 97 (2018) no.8, 083010 